\title{
Bushy pseudocharacters and group actions on quasitrees
}

\author{
ÁlVAro MARTíneZ-PÉREZ
}

Given a group acting on a graph quasi-isometric to a tree, we give sufficient conditions for a pseudocharacter to be bushy. We relate this with the conditions studied by Bestvina and Fujiwara on their work on bounded cohomology and obtain some results on the space of pseudocharacters.

57M07, 05C05; 20J06

\section{Introduction}

If $G$ is a finitely presented group, then $f: G \rightarrow \mathbb{R}$ is a quasihomomorphism or quasicharacter if $f(\alpha)+f(\beta)-f(\alpha \beta)$ is bounded independent of $\alpha, \beta$.

If $G$ is a finitely presented group, then $f: G \rightarrow \mathbb{R}$ is a pseudocharacter if it has the following properties:

- $f\left(\alpha^{n}\right)=n \alpha$ for all $\alpha \in G, n \in \mathbb{Z}$.

- $\delta f(\alpha, \beta)=f(\alpha)+f(\beta)-f(\alpha \beta)$ is bounded independent of $\alpha, \beta$.

Clearly the constant map $f(G)=0$ is a trivial pseudocharacter.

Remark Note that if $f$ is a quasicharacter and $\phi$ is given by

$$
\phi(g)=\lim _{n \rightarrow \infty} f\left(g^{n}\right) / n,
$$

then $\phi$ is a pseudocharacter with $\phi-f$ bounded.

Let $S$ be a finite generating set for $G$. If $\Gamma(G, S)$ is the Cayley graph associated to the generating set $S$, then $f$ can be extended affinely over the edges of $\Gamma(G, S)$.

If $\phi: \mathbb{R}_{+} \rightarrow \Gamma(G, S)$ is an infinite ray, then the sign of $\phi$ is

$$
\sigma(\phi)= \begin{cases}+1 & \text { if } \lim _{t \rightarrow \infty} f \circ \phi(t)=\infty, \\ -1 & \text { if } \lim _{t \rightarrow \infty} f \circ \phi(t)=-\infty, \\ 0 & \text { otherwise }\end{cases}
$$


If $w$ is some infinite word in the generators $S$, there is a path $\phi_{w}: \mathbb{R}_{+} \rightarrow \Gamma(G, S)$ beginning at 1 and realizing the word. Define $\sigma(w)=\sigma\left(\phi_{w}\right)$. If $g$ is a group element, let $\sigma(g)$ be the sign of $f(g)$. Notice that if we pick a word $w$ representing $g$ then $\sigma(w w w \cdots)=\sigma\left(w^{\infty}\right)=\sigma(g)$.

\section{Definition 1.1 Let}

$$
E(f, S)=\left\{w=w_{1} w_{2} \cdots \mid w_{i} \in S \cup S^{-1} \text { and } \sigma(w) \in\{+1,-1\}\right\} / \sim,
$$

where $w=w_{1} w_{2} \cdots \sim_{C} v=v_{1} v_{2} \cdots$ if $\sigma(w)=\sigma(v)$ and for all $D$ with $\sigma(w) D>C$ there is a word $d=d_{1} \cdots d_{n}$ in the letters $S \cup S^{-1}$ such that:

- $w_{p} d=v_{p}$ in $G$ for some prefix $w_{p}$ of $w$ and some prefix $v_{p}$ of $v$,

- $\left|f\left(w_{p} d_{p}\right)-D\right| \leq C$ for all prefixes $d_{p}$ of $d$.

The word $d$ will be referred to as a connecting word and $w \sim v$ if $w \sim_{C} v$ for some $C$. This is an equivalence relation.

Since the set $E(f, S)$ is invariant under change of generators (see Manning [12, 2.3]) it can be denoted just by $E(f)$.

Let $f: G \rightarrow \mathbb{R}$ be a pseudocharacter. $E(f)^{+} \subset E(f)$ denotes the set of positive elements of $E(f)$, and $E(f)^{-} \subset E(f)$ the set of negative elements. If $|E(f)|=2$, $f$ is said to be uniform. If $\left|E(f)^{+}\right|=1$ or $\left|E(f)^{-}\right|=1$ but $f$ is not uniform, then $f$ is said to be unipotent. Otherwise, $f$ is said to be bushy.

This work is mainly based in two papers. The first is due to Bestvina and Fujiwara [2]. In the first part of that work they consider a group acting on a $\delta$-hyperbolic graph by isometries. There, they finish the work started by Fujiwara in $[5 ; 6 ; 7]$ proving that if the action holds certain conditions (Manning called this a Bestvina-Fujiwara action), then the dimension of the second bounded cohomology of $G$ as a vector space over $\mathbb{R}$ is the cardinal of the continuum.

On the other hand, Manning proves in [12] two interesting results about pseudocharacters. In the first one the author proves that if for a given group $G$ there is a nonuniform pseudocharacter, then $G$ admits a cobounded quasiaction on a bushy tree. To do that, he also defines Bottleneck Property characterizing when a metric space is quasi-isometric to a tree.

The second one relates the existence of a bushy pseudocharacter with the conditions on the action studied in [2].

Proposition $1.2[12,4.27]$ If $f: G \rightarrow \mathbb{R}$ is a bushy pseudocharacter, then there is a Bestvina-Fujiwara action of $G$ on a quasitree. 
Herein we work in the opposite direction. In Section 2, we give some sufficient conditions for the existence of nonuniform pseudocharacters.

Proposition 2.7 Let $G$ be a group acting on a quasitree $X$. Let $g_{1}, g_{2}$ be two hyperbolic elements of $G$ such that $d_{H}\left(\Gamma_{1}\left(g_{1}, x_{0}, \gamma_{1}\right), \Gamma_{2}\left(g_{2}, x_{0}, \gamma_{2}\right)\right)=\infty$. Then, if $h$ is a pseudocharacter such that $h\left(g_{1}\right)>0$ and $h\left(g_{2}\right)>0$ and $h$ is bornologous on the action then $g_{1}^{\infty} \not g_{2}^{\infty}$ in $E(h)$.

Corollary 2.11 Let $G$ be a group acting by isometries on a quasitree $X$ so that the action is metrically proper. Let $g_{1}, g_{2}$ be two hyperbolic elements of $G$ such that $d_{H}\left(\Gamma_{1}\left(g_{1}, x_{0}, \gamma_{1}\right), \Gamma_{2}\left(g_{2}, x_{0}, \gamma_{2}\right)\right)=\infty$. Then, if $h$ is a pseudocharacter such that $h\left(g_{1}\right)>0$ and $h\left(g_{2}\right)>0$ then $g_{1}^{\infty} \nsim g_{2}^{\infty}$ in $E(h)$.

Corollary 2.15 Consider a nonelementary action of a group $G$ on a quasitree $X$. If the action is metrically proper then every nonelementary pseudocharacter is bushy.

In Section 3 we prove that given a Bestvina-Fujiwara action, it holds the conditions of Proposition 2.7. Moreover:

Theorem 3.10 Let $G$ be a group acting on a quasitree. If it is a Bestvina-Fujiwara action, then there is a bushy pseudocharacter $h: G \rightarrow \mathbb{R}$.

Examples of Bestvina-Fujiwara actions on quasitrees may be built using some axiomatic construction defined by Bestvina, Bromberg and Fujiwara in [1]. We include in this section a short explanation of how that works.

A quasiaction of a group $G$ on a metric space $X$ associates to each $g \in G$ a quasiisometry $A_{g}: X \rightarrow X$ with uniform quasi-isometry constants so that $A_{\mathrm{Id}}=\operatorname{Id}_{X}$ and such that the distance between $A_{h} \circ A_{g}$ and $A_{h g}$ in the sup norm is uniformly bounded independent of $g, h \in G$. This is a natural and interesting extension of group actions and it has been relevant in relation to trees. In [15], Mosher, Sageev, and Whyte prove that every cobounded quasiaction on a bounded valence bushy tree is quasiconjugate to an action on a tree. However, there are examples of quasiactions on simplicial trees which are not quasiconjugate to actions on $\mathbb{R}$-trees. See [12] for the examples and [13] for further results on quasiactions on trees.

Given a nonuniform pseudocharacter $h$, Manning builds in [12] a cobounded quasiaction on a bushy tree $T$. In Section 4 we show that this construction can be made by adding a condition to the relation between the space $E(h)$ and the boundary of the tree $\partial T$.

In the last section we state some implications on the space of pseudocharacters and therefore, in the cobounded cohomology of the group. 
Corollary 5.4 If there is a bushy pseudocharacter $h: G \rightarrow \mathbb{R}$ then the dimension of the space generated by the bushy pseudocharacters on $G$ as a vector space over $\mathbb{R}$ is the cardinal of the continuum.

All groups are assumed to be finitely presented.

Acknowledgements The author would like to express his gratitude to the mathematics department of the UIC for their hospitality and to Kevin Whyte for suggesting this work. The author would like also to thank the referee and Bestvina for their helpful comments which significantly improved this manuscript. The author is partially supported by MTM-2009-07030.

\section{Actions and pseudocharacters}

Definition 2.1 A map between metric spaces, $f:\left(X, d_{X}\right) \rightarrow\left(Y, d_{Y}\right)$, is said to be quasi-isometric if there are constants $\lambda \geq 1$ and $C>0$ such that for all $x, x^{\prime} \in X$, $(1 / \lambda) d_{X}\left(x, x^{\prime}\right)-A \leq d_{Y}\left(f(x), f\left(x^{\prime}\right)\right) \leq \lambda d_{X}\left(x, x^{\prime}\right)+A$. If there is a constant $B>0$ such that $N_{B}(f(X))=Y$ where $N_{B}(f(X))=\left\{y \in Y \mid d_{Y}(y, f(X))<B\right\}$, then $f$ is a quasi-isometry and $X, Y$ are quasi-isometric.

Theorem 2.2 [12, Theorem 4.6] Let $Y$ be a geodesic metric space. The following are equivalent:

(1) $Y$ is quasi-isometric to some simplicial tree $\Gamma$.

(2) (Bottleneck Property) There is some $\Delta>0$ so that for all $x, y \in Y$ there is a midpoint $m=m(x, y)$ with $d(x, m)=d(y, m)=\frac{1}{2} d(x, y)$ and the property that any path from $x$ to $y$ must pass within less than $\Delta$ of the point $m$.

Let $(X, d)$ be a metric space. Fix a base point $o \in X$ and for $x, x^{\prime} \in X$ put $\left(x \mid x^{\prime}\right)_{o}=$ $\frac{1}{2}\left(d(x, o)+d\left(x^{\prime}, o\right)-d\left(x, x^{\prime}\right)\right)$. The number $\left(x \mid x^{\prime}\right)_{o}$ is nonnegative and it is called the Gromov product of $x, x^{\prime}$ with respect to $o$. See Gromov [9].

Definition 2.3 A metric space $X$ is (Gromov) hyperbolic if it satisfies the $\delta$-inequality

$$
(x \mid y)_{o} \geq \min \left\{(x \mid z)_{o},(z \mid y)_{o}\right\}-\delta
$$

for some $\delta \geq 0$, for every base point $o \in X$ and all $x, y, z \in X$. 
Let $X$ be a hyperbolic space and $o \in X$ a base point. A sequence of points $\left\{x_{i}\right\} \subset X$ converges to infinity if

$$
\lim _{i, j \rightarrow \infty}\left(x_{i} \mid x_{j}\right)_{o}=\infty
$$

This property is independent of the choice of $o$ since

$$
\left|\left(x \mid x^{\prime}\right)_{o}-\left(x \mid x^{\prime}\right)_{o^{\prime}}\right| \leq\left|o o^{\prime}\right|
$$

for any $x, x^{\prime}, o, o^{\prime} \in X$.

Two sequences $\left\{x_{i}\right\},\left\{x_{i}^{\prime}\right\}$ that converge to infinity are equivalent if

$$
\lim _{i \rightarrow \infty}\left(x_{i} \mid x_{i}^{\prime}\right)_{o}=\infty \text {. }
$$

Using the $\delta$-inequality, we easily see that this defines an equivalence relation for sequences in $X$ converging to infinity. The boundary at infinity $\partial_{\infty} X$ of $X$ is defined to be the set of equivalence classes of sequences converging to infinity.

The following lemma is a well known property of quasigeodesics (see Bowditch [3] or Fujiwara [6]). The statement with the proof can be found in Manning [13].

Lemma 2.4 Let $X$ be a $\delta$-hyperbolic space. Given $K \geq 1$ and $L \geq 0$, there exists $B(K, L, \delta) \geq 0$ such that if $\gamma_{1}, \gamma_{2}$ are two $(K, L)$-quasigeodesics with the same endpoints in $X \cup \partial X$, then $\gamma_{1} \subset N_{B}\left(\gamma_{2}\right)$ and $\gamma_{2} \subset N_{B}\left(\gamma_{1}\right)$.

Definition 2.5 Fix $x_{0} \in X$, where $X$ is a $\delta$-hyperbolic metric space on which $G$ quasiacts. Let $O_{g, x}: \mathbb{R} \rightarrow X$ be defined by $O_{g, x}(t)=g^{\lfloor t\rfloor} x$ where $\lfloor t\rfloor$ is the largest integer smaller than $t$. Then it is said that $g$ quasiacts elliptically if $O_{g, x}$ has bounded image, and $g$ quasiacts hyperbolically if $O_{g, x}$ is a quasigeodesic. If $G$ acts isometrically on $X$ then it is said that $g$ acts elliptically or hyperbolically or that $g$ is elliptic or hyperbolic.

It is readily seen that this definition is independent of $x$ and agrees with the standard definitions in case $G$ acts isometrically.

If $g \in G$ is hyperbolic $x \in X$, and $\gamma_{0}:[0,1] \rightarrow X$ is a geodesic segment with $\gamma_{0}(0)=x$ and $\gamma_{0}(1)=g(x)$, then it is not hard to check that $\Gamma_{g, x, \gamma_{0}}: \mathbb{R} \rightarrow X$ given by

$$
\Gamma_{g, x, \gamma_{0}}(t)=g^{\lfloor t\rfloor} \gamma_{0}(t-\lfloor t\rfloor)
$$

is a continuous quasigeodesic. Moreover, $g$ is an isometry of $X$ which maps this quasigeodesic to itself by a nontrivial translation. See Figure 1.

A quasigeodesic where $g$ acts by nontrivial translation will be referred to as a quasiaxis (or $(K-L)$-quasiaxis if the constants are relevant). A quasiaxis of $g$ is given the $g$-orientation by the requirement that $g$ acts as a positive translation. 


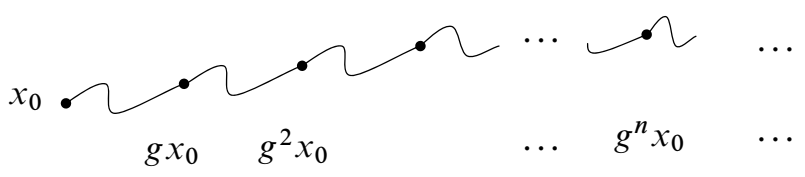

Figure 1: The isometry $g$ acts on $\Gamma_{g, x, \gamma_{0}}$ by a nontrivial translation.

Definition 2.6 Let $G$ be a group acting by isometries on a metric space $X$. We say that a pseudocharacter $h: G \rightarrow \mathbb{R}$ is bornologous on the action if given any $x_{0} \in X$ and any $g \in G$, for all $R>0$ there exists $S>0$ such that for all $g^{\prime} \in G$ with $g^{\prime}\left(x_{0}\right) \in B\left(g\left(x_{0}\right), R\right),\left|h\left(g^{\prime}\right)-h(g)\right| \leq S$.

A quasitree is a complete geodesic metric space quasi-isometric to some simplicial tree. These spaces satisfy bottleneck property; see Theorem 2.2. Herein, we will add the assumption that the quasitree is a graph. This is not a restrictive assumption since we are working in a coarse setting but it has obvious technical advantages. Therefore, from now on, a quasitree will be a graph satisfying bottleneck property.

Proposition 2.7 Let $G$ be a group acting on a quasitree $X$. Let $g_{1}, g_{2}$ be two hyperbolic elements of $G$ such that $d_{H}\left(\Gamma_{1}\left(g_{1}, x_{0}, \gamma_{1}\right), \Gamma_{2}\left(g_{2}, x_{0}, \gamma_{2}\right)\right)=\infty$. Then, if $h$ is a pseudocharacter such that $h\left(g_{1}\right)>0$ and $h\left(g_{2}\right)>0$ and $h$ is bornologous on the action then $g_{1}^{\infty} \not g_{2}^{\infty}$ in $E(h)$.

Proof Since $h\left(g_{1}\right)>0$ and $h\left(g_{2}\right)>0, \sigma_{h}\left(g_{1}\right)>0$ and $\sigma_{h}\left(g_{2}\right)>0$. Let $w_{j}$ be the word representing $g_{j}$ in the letters of the generating set $S \cup S^{-1}$. Then $w_{j} w_{j} \cdots=w_{j}^{\infty}$ is an element of $E(h)$ fixed by $g_{j}$ for $j=1,2$. Note that, with the assumptions taken, $\sigma\left(w_{j}^{\infty}\right)=+1$.

Let us see that $w_{1}^{\infty} \nsucc w_{2}^{\infty}$ in $E(h)$.

Let us denote, for simplicity, $w=w_{1}^{\infty}$ and $v=w_{2}^{\infty}$ and suppose $w \sim_{C} v$ for some $C>0$. Then, given $D_{1}>C$ there is a connecting word $d=d_{1} \cdots d_{n}$ such that:

- $w_{p} d=v_{p}$ in $G$ for some prefix $w_{p}$ of $w$ and some prefix $v_{p}$ of $v$,

- $\left|h\left(w_{p} d_{p}\right)-D_{1}\right| \leq C$ for all prefixes $d_{p}$ of $d$.

By abuse of notation let us identify the group element $g$ with the word representing it, $w$. Therefore, we write $w\left(x_{0}\right)$ for the image of the isometric action $g$ on $x_{0}$. Let $\gamma$ be a geodesic path from $w_{p}\left(x_{0}\right)$ to $v_{p}\left(x_{0}\right)$ and let $m$ be the midpoint in $\gamma$.

Let $\gamma_{1}$ be a geodesic path from $x_{0}$ to $w_{1}\left(x_{0}\right)$ and $\gamma_{2}$ be a geodesic path from $x_{0}$ to $w_{2}\left(x_{0}\right)$ and consider $\Gamma_{1}:=\Gamma_{g_{1}, x_{0}, \gamma_{1}}(t), \Gamma_{2}:=\Gamma_{g_{2}, x_{0}, \gamma_{2}}(t)$ two continuous quasigeodesics defined as in (1). Let $\Gamma_{1}\left(w_{p}, w_{q}\right)$ be the restriction of $\Gamma_{g_{1}, x_{0}, \gamma_{1}}(t)$ to a 
(quasi-isometric) path from $w_{p}\left(x_{0}\right)$ to $w_{q}\left(x_{0}\right)$ for any prefixes $w_{p}, w_{q}$ of $w$. Also, let $\Gamma_{2}\left(v_{p}, v_{q}\right)$ be the restriction of $\Gamma_{g_{2}, x_{0}, \gamma_{2}}(t)$ to a (quasi-isometric) path from $v_{p}\left(x_{0}\right)$ to $v_{q}\left(x_{0}\right)$ for any prefixes $v_{p}, v_{q}$ of $v$.

Let $\Delta$ be the bottleneck property constant.

Claim Since $d_{H}\left(\Gamma_{1}\left(g_{1}, x_{0}, \gamma_{1}\right), \Gamma_{2}\left(g_{2}, x_{0}, \gamma_{2}\right)\right)=\infty$, we may assume $D_{1}$ big enough to assure that for any $w_{p} \subset w_{q}$ and $v_{p} \subset v_{q}$, then $\Gamma_{1}\left(w_{p}, w_{q}\right) \cap B(m, \Delta)=\varnothing$ and $\Gamma_{2}\left(v_{p}, v_{q}\right) \cap B(m, \Delta)=\varnothing$. See Figure 2 .

Since $d_{H}\left(\Gamma_{1}\left(g_{1}, x_{0}, \gamma_{1}\right), \Gamma_{2}\left(g_{2}, x_{0}, \gamma_{2}\right)\right)=\infty$, we assume $D_{1}=D_{1}\left(h, \Delta, g_{1}, g_{2}\right)$ is big enough to guarantee that $A:=d\left(w_{p}, v_{p}\right)$ is as big as we want. Notice that $d\left(w_{1}^{i}\left(x_{0}\right), w_{1}^{i+1}\left(x_{0}\right)\right)=d\left(x_{0}, w_{1}\left(x_{0}\right)\right)=: d_{1}$ so $d\left(w_{1}^{i}\left(x_{0}\right), w_{1}^{i+k}\left(x_{0}\right)\right) \leq k \cdot d_{1}$. Respectively, for $w_{2}$, we have $d\left(w_{2}^{i}\left(x_{0}\right), w_{2}^{i+1}\left(x_{0}\right)\right)=d\left(x_{0}, w_{2}\left(x_{0}\right)\right)=: d_{2}$ therefore $d\left(w_{2}^{i}\left(x_{0}\right), w_{2}^{i+k}\left(x_{0}\right)\right) \leq k \cdot d_{1}$. So, if $A$ is big enough, either $k$ is also big enough (depending on the distance $\left.(A / 2-\Delta) / \max \left\{d_{1}, d_{2}\right\}\right)$ or we can assure the quasigeodesic $\Gamma_{1}\left(w_{p}, w_{p} \cdot w_{1}^{k}\right)$ from $w_{p}\left(x_{0}\right)$ to $w_{p} \cdot w_{1}^{k}\left(x_{0}\right)$ satisfies $\Gamma_{1}\left(w_{p}, w_{p} \cdot w_{1}^{k}\right) \cap B(m, \Delta)=\varnothing$. Also, either $k$ is big enough or the quasigeodesic $\Gamma_{2}\left(v_{p}, v_{p} \cdot w_{2}^{k}\right)$ from $v_{p}\left(x_{0}\right)$ to $v_{p} \cdot w_{2}^{k}\left(x_{0}\right)$ satisfies $\Gamma_{2}\left(v_{p}, v_{p} \cdot w_{2}^{k}\right) \cap B(m, \Delta)=\varnothing$.

Now, let us assume $k=k\left(h, \Delta, g_{1}, g_{2}\right)$ as big as we want and fix it assuming that the corresponding quasigeodesics $\Gamma_{1}\left(w_{p}, w_{p} \cdot w_{1}^{k}\right)$ and $\Gamma_{2}\left(v_{p}, v_{p} \cdot w_{2}^{k}\right)$ do not intersect the ball $B(m, \Delta)$. By hypothesis we have that $h\left(g_{j}\right)>0$ and $h\left(g_{j}^{n}\right)=n h\left(g_{j}\right)$ for $j=1,2$. Then, for any $j \geq k$, we have $h\left(w_{p} \cdot w_{1}^{j}\left(x_{0}\right)\right)=h\left(w_{p}\right)+j h\left(g_{1}\right)$ and $h\left(v_{p} \cdot w_{2}^{j}\right)=h\left(v_{p}\right)+j h\left(g_{2}\right)$ are much bigger than $D_{1}$. Then, since $h$ is bornologous on the action, we can assume $k$ big enough so that $w_{p} \cdot w_{1}^{j}\left(x_{0}\right) \notin B\left(m, \Delta+\max \left\{d_{1}, d_{2}\right\}\right)$ and $v_{p} \cdot w_{2}^{j}\left(x_{0}\right) \notin B\left(m, \Delta+\max \left\{d_{1}, d_{2}\right\}\right)$ for any $j \geq k$. So, the quasigeodesic segments $\Gamma_{1}\left(w_{p} \cdot w_{1}^{j}, w_{p} \cdot w_{1}^{j+1}\right) \cap B(m, \Delta)=\varnothing$ and $\Gamma_{2}\left(v_{p} \cdot w_{2}^{j}, v_{p} \cdot w_{2}^{j+1}\right) \cap B(m, \Delta)=\varnothing$ for every $j \geq 0$, proving the claim.

Now, let $D_{2}>h(m), D_{1}$. Then, we will reach a contradiction finding a uniform bound for $D_{2}-D_{1}$.

By assumption, there is a connecting word $d^{\prime}=d_{1}^{\prime} \cdots d_{n}^{\prime}$ such that:

- $w_{p^{\prime}} d^{\prime}=v_{p^{\prime}}$ in $G$ for some prefix $w_{p^{\prime}}$ of $w$ and some prefix $v_{p^{\prime}}$ of $v$,

- $\left|h\left(w_{p^{\prime}} d_{p^{\prime}}^{\prime}\right)-D_{2}\right| \leq C$ for all prefixes $d_{p^{\prime}}^{\prime}$ of $d$.

Let $\Gamma_{1}:=\Gamma_{1}\left(w_{p}, w_{p}^{\prime}\right), \Gamma_{2}:=\Gamma_{2}\left(v_{p}, v_{p}^{\prime}\right)$ be quasigeodesic paths defined as above from $w_{p}\left(x_{0}\right)$ to $w_{p^{\prime}}\left(x_{0}\right)$ and from $v_{p}\left(x_{0}\right)$ to $v_{p^{\prime}}\left(x_{0}\right)$. Let $\gamma_{j}^{\prime}:[0,1] \rightarrow X$ be a geodesic path from $w_{p^{\prime}} \cdot d_{p^{\prime}}^{\prime} \cdot d_{j-1}^{\prime}\left(x_{0}\right)$ to $w_{p^{\prime}} \cdot d_{p^{\prime}}^{\prime} \cdot d_{j}^{\prime}\left(x_{0}\right)$ for $1 \leq j \leq n$ and let $\gamma^{\prime}:[0, n] \rightarrow X$ be the path from $w_{p^{\prime}}\left(x_{0}\right)$ to $v_{p^{\prime}}\left(x_{0}\right)$ defined by $d^{\prime}$ where $\gamma^{\prime}(t)=\gamma_{\lfloor t\rfloor}^{\prime}(t-\lfloor t\rfloor)$. 


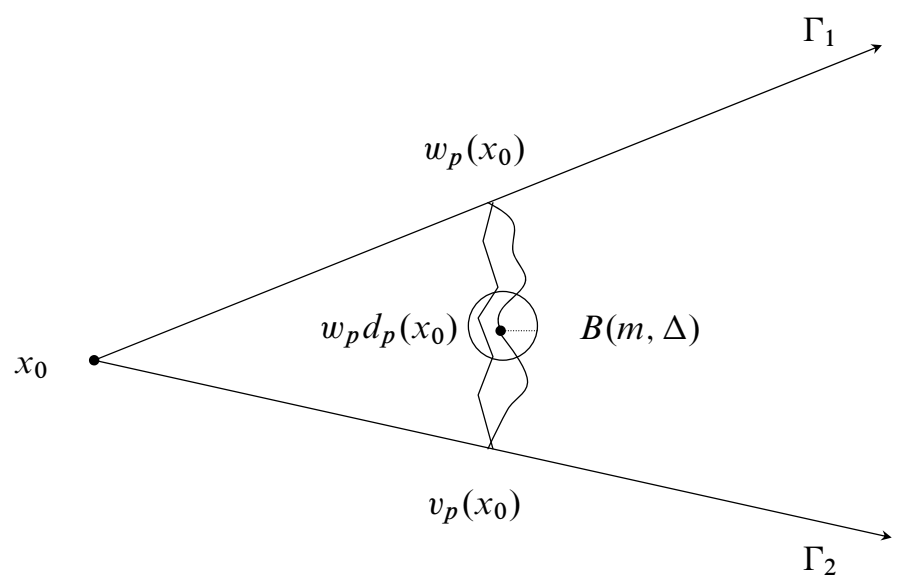

Figure 2: Assuming $D_{1}$ big enough, we obtain that $\Gamma_{1}\left(w_{p}, w_{p} \cdot w_{1}^{k}\right)$ and $\Gamma_{2}\left(v_{p}, v_{p} \cdot w_{2}^{k}\right)$ do not intersect the ball $B(m, \Delta)$.

Then, $\Gamma=\Gamma_{1} \cup \Gamma^{\prime} \cup \Gamma_{2}^{-1}$ is a path from $w_{p}\left(x_{0}\right)$ to $v_{p}\left(x_{0}\right)$. By bottleneck property (see Theorem 2.2) there is a point $x \in \Gamma$ such that $d(x, m) \leq \Delta$. By the previous claim, we can assume that $x \in \gamma_{j}^{\prime}[0,1]$ for some $1 \leq j \leq n$. See Figure 3 .

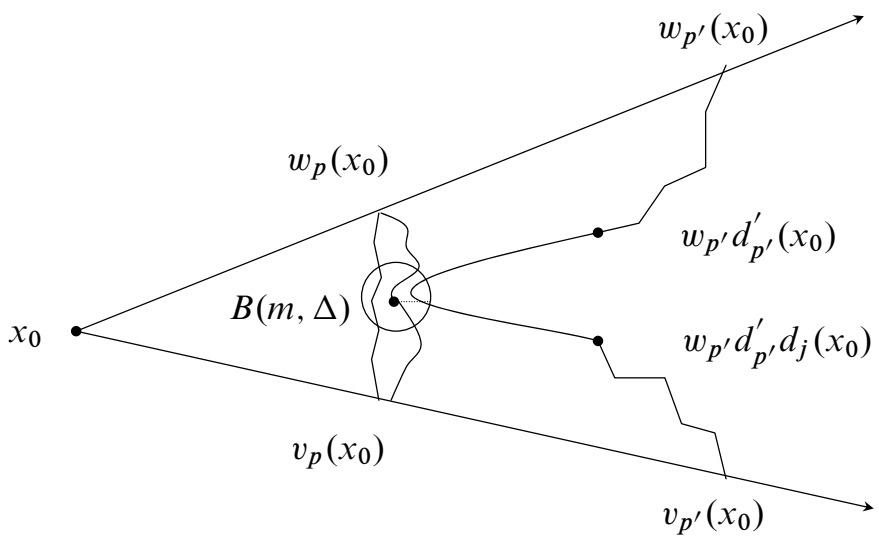

Figure 3: The path $\left[w_{p}\left(x_{0}\right), w_{p^{\prime}}\left(x_{0}\right)\right] \cup\left[w_{p^{\prime}}\left(x_{0}\right), v_{p^{\prime}}\left(x_{0}\right)\right] \cup\left[v_{p^{\prime}}\left(x_{0}\right), v_{p}\left(x_{0}\right)\right]$ intersects the ball $B(m, \Delta)$ by bottleneck property.

By hypothesis, $h\left(w_{p^{\prime}} d_{p^{\prime}}^{\prime}\right), h\left(w_{p^{\prime}} d_{p^{\prime}}^{\prime} d_{j}^{\prime}\right) \in\left(D_{2}-C, D_{2}+C\right)$. Let $F=F\left(S, x_{0}\right)$ be a constant so for all $s \in S, d\left(x_{0}, s\left(x_{0}\right)\right) \leq F$. Then, $d\left(w_{p^{\prime}} d_{p^{\prime}}^{\prime}\left(x_{0}\right), w_{p^{\prime}} d_{p^{\prime}}^{\prime} d_{j}^{\prime}\left(x_{0}\right)\right) \leq F$. Therefore, $d\left(w_{p^{\prime}} d_{p^{\prime}}^{\prime}\left(x_{0}\right), m\right) \leq \Delta+F$ and $d\left(w_{p} d_{p}^{\prime} d_{j}^{\prime}\left(x_{0}\right), m\right) \leq \Delta+F$ which implies $d\left(w_{p^{\prime}} d_{p^{\prime}}^{\prime}\left(x_{0}\right), w_{p} d_{p}\left(x_{0}\right)\right) \leq 2 \Delta+2 F$ and that $d\left(w_{p} d_{p}^{\prime} d_{j}^{\prime}\left(x_{0}\right), w_{p} d_{p}\left(x_{0}\right)\right) \leq 2 \Delta+2 F$ for some prefix $d_{p}$ of $d$. But since $h$ is bornologous on the action, there is some $S=S(2 \Delta+2 F)$ such that $\left|h\left(w_{p} d_{p}\right)-h\left(w_{p} d_{p}^{\prime} d_{j}^{\prime}\right)\right|<S$ and $D_{2}-D_{1}<S+2 C$. 
Thus, $D_{2}-D_{1}$ is bounded by a constant depending only on $h, C, F, \gamma_{1}, \gamma_{2}$ and $\Delta$, leading to contradiction.

Definition 2.8 An action of a group by isometries on a metric space is metrically proper if for all $x \in X$ and for all $R>0$ the set $\{g \in G \mid g(N(x, R)) \cap N(x, R) \neq \varnothing\}$ is finite.

Lemma 2.9 An action of a group $G$ by isometries on a metric space $X$ is metrically proper if and only if for all $x_{0} \in X$ and for all $g \in G$ then for all $R>0$ the set $\left\{g^{\prime} \in G \mid g^{\prime} x_{0} \in N\left(g\left(x_{0}\right), R\right)\right\}$ is finite.

Proof The set $\left\{h \in G \mid h\left(B\left(g\left(x_{0}\right), R\right)\right) \cap B\left(g\left(x_{0}\right), R\right) \neq \varnothing\right\}$ is finite if the action is metrically proper. If $g^{\prime}=h g$, then $\left\{g^{\prime} \in G \mid g^{\prime} g^{-1} B\left(g\left(x_{0}\right), R\right) \cap B\left(g\left(x_{0}\right), R\right) \neq \varnothing\right\}=$ $\left\{g^{\prime} \in G \mid g^{\prime} B\left(x_{0}, R\right) \cap B\left(g\left(x_{0}\right), R\right) \neq \varnothing\right\}$ is finite. In particular, the set $\left\{g^{\prime} \in G \mid\right.$ $\left.g^{\prime} x_{0} \in B\left(g\left(x_{0}\right), R\right)\right\}$ is finite.

Conversely, suppose $\left\{g^{\prime} \in G \mid g^{\prime} x_{0} \in N\left(g\left(x_{0}\right), 2 R\right)\right\}$ is finite. Then, if $h=g^{\prime} g^{-1}$, the set $\left.\left.\left\{h \in G \mid(h g)\left(x_{0}\right) \cap N\left(g\left(x_{0}\right), 2 R\right)\right) \neq \varnothing\right\}=\left\{h \in G \mid h\left(g\left(x_{0}\right)\right) \cap N\left(g\left(x_{0}\right), 2 R\right)\right) \neq \varnothing\right\}$ is finite which implies $\left.\left\{h \in G \mid h\left(N\left(g\left(x_{0}\right), R\right)\right) \cap N\left(g\left(x_{0}\right), R\right)\right) \neq \varnothing\right\}$ is finite.

Proposition 2.10 Let $G$ be a group acting by isometries on a metric space $X$ and let $h: G \rightarrow \mathbb{R}$ be a pseudocharacter. If the action is metrically proper then the pseudocharacter is bornologous on the action.

Proof Let $x_{0} \in X, g \in G$ and $R>0$. Since the action is metrically proper the set $K=\left\{g^{\prime} \in G \mid g^{\prime} x_{0} \in N\left(g\left(x_{0}\right), R\right)\right\}$ is finite. Therefore, it suffices to take $S:=\max _{g^{\prime} \in K}\left\{\left|h\left(g^{\prime}\right)-h(g)\right|\right\}$.

Corollary 2.11 Let $G$ be a group acting by isometries on a quasitree $X$ so that the action is metrically proper. Let $g_{1}, g_{2}$ be two hyperbolic elements of $G$ such that $d_{H}\left(\Gamma_{1}\left(g_{1}, x_{0}, \gamma_{1}\right), \Gamma_{2}\left(g_{2}, x_{0}, \gamma_{2}\right)\right)=\infty$. Then, if $h$ is a pseudocharacter such that $h\left(g_{1}\right)>0$ and $h\left(g_{2}\right)>0$ then $g_{1}^{\infty} \nsim g_{2}^{\infty}$ in $E(h)$.

Definition 2.12 Two hyperbolic isometries $g_{1}, g_{2}$ are said to be independent if their quasiaxis do not contain rays which are a finite Hausdorff distance apart. Equivalently the fixed point sets of $g_{1}, g_{2}$ in $\partial X$ are disjoint. An action is nonelementary if there are at least two independent hyperbolic elements.

Definition 2.13 We say that a pseudocharacter $h: G \rightarrow \mathbb{R}$ is nonelementary if there is a pair of independent $g_{1}, g_{2} \in G$ such that $h\left(g_{1}\right) \neq 0$ and $h\left(g_{2}\right) \neq 0$. 
Corollary 2.14 Consider a nonelementary action of a group $G$ on a quasitree $X$ and a nonelementary pseudocharacter $h: G \rightarrow \mathbb{R}$. Then, if $h$ is bornologous on the action, it is bushy.

Corollary 2.15 Consider a nonelementary action of a group $G$ on a quasitree $X$. If the action is metrically proper then every nonelementary pseudocharacter is bushy.

\section{Existence of bushy pseudocharacters}

Note any two $(K-L)$-quasiaxis of $g$ are within some universal $B=B(\delta, K, L)$ of one another and any sufficiently long $(K, L)$-quasigeodesic arc $J$ in a $B$-neighborhood of a quasiaxis $l$ of $g$ inherits a natural $g$-orientation: a point of $l$ within $B(\delta, K, L)$ of the terminal endpoint of $J$ is ahead (with respect to the $g$-orientation of $l$ ) of a point of $l$ within $B(\delta, K, L)$ of the initial endpoint of $J$. This orientation of $J$ will be denoted by $g$-orientation of $J$.

Definition 3.1 (Bestvina and Fujiwara [2]) If $g_{1}, g_{2}$ are hyperbolic elements of $G$, let $g_{1} \sim g_{2}$ if for an arbitrarily long segment $J$ in a $(K, L)$-quasiaxis for $g_{1}$ there is a $g \in G$ such that $g(J)$ is within $B(\delta, K, L)$ of a $(K, L)$-quasiaxis of $g_{2}$ and $g: J \rightarrow g(J)$ is orientation-preserving with respect to the $g_{2}$-orientation on $g(J)$.

This defines an equivalence relation. As it is said in [2], the concept does not change if $B$ is replaced by a larger constant.

Definition 3.2 A Bestvina-Fujiwara action is a nonelementary action of a group $G$ on a hyperbolic graph $X$ so that there exist independent $g_{1}, g_{2}$ such that $g_{1} \not g_{2}$.

Lemma 3.3 Let $X$ be a (geodesic) Gromov hyperbolic space and let $G$ be a group acting by isometries on $X$. Let $g_{1}, g_{2} \in G$ such that $g_{1} \not g_{2}$. Then, for any point $x_{0} \in X$, and any pair of geodesics $\gamma_{1}:=\left[x_{0}, g_{1}\left(x_{0}\right)\right], \gamma_{2}:=\left[x_{0}, g_{2}\left(x_{0}\right)\right]$, $d_{H}\left(\Gamma_{1}\left(g_{1}, x_{0}, \gamma_{1}\right), \Gamma_{2}\left(g_{2}, x_{0}, \gamma_{2}\right)\right)=\infty$.

Proof Suppose there is some $B>0$ such that $\Gamma_{1} \subset N_{B}\left(\Gamma_{2}\right)$ and $\Gamma_{2} \subset N_{B}\left(\Gamma_{1}\right)$. Then, the action of the identity $i$ on $\Gamma_{1}$ is contained in $N_{B}\left(\Gamma_{2}\right)$ and $i: \Gamma_{1} \rightarrow i\left(\Gamma_{1}\right)=\Gamma_{1}$ is orientation preserving with respect to the $g_{1}$-orientation and the $g_{2}$-orientation on $\Gamma_{1}$. This contradicts the fact that $g_{1} \not g_{2}$. 
Let us recall now the basic construction of quasihomomorphisms associated to the action as presented in [6].

Let $X$ be a hyperbolic graph and a group $G$ acting on $X$. Let $w$ be a finite (oriented) path in $X$. By $|w|$ denote the length of $w$, by $i(w)$ the starting point and by $t(w)$ the finishing point. For $g \in G, g \circ w$ is a path starting at $g(i(w))$ and finishing at $g(t(w))$ and it is called a copy of $w$. Obviously, $|g \circ w|=|w|$.

Let $\alpha$ be a finite path. Define

$$
|\alpha|_{w}=\{\text { the maximal number of nonoverlapping copies of } w \text { in } \alpha\} .
$$

Suppose $x, y \in X$ are two vertices and that $W$ is an integer with $0<W<|w|$. Then

$$
c_{w, W}(x, y)=d(x, y)-\inf _{\alpha}\left(|\alpha|-W|\alpha|_{w}\right),
$$

where $\alpha$ ranges over all paths from $x$ to $y$.

Lemma 3.4 [6, Lemma 3.4] Let $x, y, z$ be three points in $X$. Then

$$
\left|c_{w, W}(x, y)-c_{w, W}(x, z)\right| \leq 2 d(y, z) .
$$

Let us omit $W$ from the notation and write $c_{w}$. Define $h_{w}: G \rightarrow \mathbb{R}$ by

$$
h_{w}(g)=c_{w}\left(x_{0}, g\left(x_{0}\right)\right)-c_{w^{-1}}\left(x_{0}, g\left(x_{0}\right)\right) .
$$

Proposition 3.5 [6, Proposition 3.10] If $X$ is a $\delta$-hyperbolic space $h_{w}$ is a quasihomomorphism (ie quasicharacter).

From Lemma 3.4, it is immediate to obtain the following.

Lemma 3.6 Let $X$ be a (geodesic) Gromov hyperbolic space. For any word $w$, any points $x_{0}, x \in X$ and any constants $0<W<|w|, R>0$, the subset of the real line $\left\{h_{w}(g) \mid g\left(x_{0}\right) \in B(x, R)\right\}$ is bounded. In particular, it has diameter at most $8 R$.

Therefore, the following is immediate.

Proposition 3.7 Let $X$ be a (geodesic) Gromov hyperbolic space. For any word $w$, any points $x_{0}, x \in X$ and any constants $0<W<|w|, h_{w}$ is bornologous on the action.

Let us recall two propositions from [2]. 
Proposition 3.8 [2, Proposition 2] Suppose a group $G$ acts on a $\delta$-hyperbolic graph $X$ by isometries. Suppose also that the action is nonelementary and that there exist independent hyperbolic elements $g_{1}, g_{2} \in G$ such that $g_{1} \not g_{2}$. Then, there is a sequence $f_{1}, f_{2}, \ldots \in G$ of hyperbolic elements such that:

- $f_{i} \nsim f_{i}^{-1}$ for $i=1,2, \ldots$,

- $f_{i} \nsim f_{j}^{ \pm 1}$ for $j<i$.

Replacing if necessary $g_{1}, g_{2}$ by high positive powers of conjugates, let $F$ be a free subgroup of $G$ with basis $\left\{g_{1}, g_{2}\right\}$ such that each nontrivial element of $F$ is hyperbolic and $F$ is quasiconvex with respect to the action on $X$. See the proof of [2, Propostion 2] and [9, Section 5.3] for details. Such free groups are called Schottky groups.

Proposition 3.9 [2, Proposition 5] Suppose $1 \neq f \in F$ is cyclically reduced and $f \not f^{-1}$. Then there is $a>0$ such that $h_{f}$ is unbounded on $\langle f\rangle$. Moreover, if $f^{ \pm 1} \not f^{\prime} \in F$ then $h_{f}$ is 0 on $\left\langle f^{\prime}\right\rangle$ for sufficiently large $a>0$.

Theorem 3.10 Let $G$ be a group acting on a quasitree. If it is a Bestvina-Fujiwara action, then there is a bushy pseudocharacter $h: G \rightarrow \mathbb{R}$.

Proof Consider the sequence $f_{1}, f_{1}, \ldots$ from Proposition 3.8 and assume in addition (without loss of generality) that each $f_{i}$ is cyclically reduced. Define $h_{i}: G \rightarrow \mathbb{R}$ as $h_{i}=h_{f} a_{i}$ where $a_{i}$ is chosen as in Proposition 3.9 so that $h_{i}$ is unbounded on $\left\langle f_{i}\right\rangle$ and so that it is 0 on $\left\langle f_{j}\right\rangle$ for $j<i$ and also 0 on $\left\langle f_{i+1}\right\rangle$. With the same argument, we may also assume that $\lim _{k \rightarrow \infty} h_{i}\left(f_{i}^{k}\right)=+\infty$.

Let $h$ be a pseudocharacter at a bounded distance (see Remark) from the quasicharacter $h_{1}+h_{2}$. (Notice that everything works if we consider $h_{i}$ and $h_{i+1}$ instead). Clearly, $h\left(f_{1}\right)>0$ and $h\left(f_{2}\right)>0$.

Thus $\sigma_{h}\left(f_{j}\right)>0$ and $f_{j}^{ \pm \infty}$ defines an element in $E(h)$ for $j=1,2$. Let $w_{j}$ be the word representing $f_{j}$ in the letters $S \cup S^{-1}$. Then $w_{j} w_{j} \cdots=w_{j}^{\infty}$ is an element of $E(f)$ fixed by $f_{j}$ for $j=1,2$. Note that, with the assumptions taken, $\sigma\left(w_{j}^{\infty}\right)=+1$.

Let us see that $w_{1}^{\infty} \nsucc w_{2}^{\infty}$ in $E(h)$. It suffices to check that by Proposition 3.7 and Lemma 3.3 we are in the conditions of Proposition 2.7.

The same argument proves that $w_{1}^{-\infty} \nsim w_{2}^{-\infty}$ in $E(h)$.

Corollary 3.11 If a Cayley graph $X=\Gamma(G, S)$ satisfies the bottleneck property and the canonical action of the group is a Bestvina-Fujiwara action, then there is a bushy pseudocharacter $h: G \rightarrow \mathbb{R}$. 
Bestvina, Bromberg and Fujiwara give in [1] a general construction of actions of groups on quasitrees. They proceed axiomatically defining a distance function and then modifying it to define what they call projection complex.

Let $\mathbf{Y}$ be a set and assume that for each $Y \in \mathbf{Y}$ there is a function

$$
d_{Y}^{\pi}:(\mathbf{Y} \backslash\{Y\}) \times(\mathbf{Y} \backslash\{Y\}) \rightarrow[0, \infty)
$$

and a constant $\xi>0$ that satisfies the following axioms:

(A1) $d_{Y}^{\pi}(X, Z)=d_{Y}^{\pi}(Z, X)$

(A2) $d_{Y}^{\pi}(X, Z)+d_{Y}^{\pi}(Z, W) \geq d_{Y}^{\pi}(X, W)$

(A3) $\min \left\{d_{Y}^{\pi}(X, Z), d_{Z}^{\pi}(X, Y)\right\}<\xi$

(A4) $\#\left\{Y \mid d_{Y}^{\pi}(X, Z) \geq \xi\right\}$ is finite for all $X, Z \in \mathbf{Y}$

Given this distance function they define $\mathcal{H}(X, Z)$ to be the set of pairs $\left(X^{\prime}, Z^{\prime}\right) \in \mathbf{Y} \times \mathbf{Y}$ such that one of the following holds:

- $d_{X}^{\pi}\left(X^{\prime}, Z^{\prime}\right), d_{Z}^{\pi}\left(X^{\prime}, Z^{\prime}\right)>2 \xi$

- $X=X^{\prime}$ and $d_{Z}^{\pi}\left(X, Z^{\prime}\right)>2 \xi$

- $Z=Z^{\prime}$ and $d_{X}^{\pi}\left(X^{\prime}, Z\right)>2 \xi$

- $\left(X^{\prime}, Z^{\prime}\right)=(X, Z)$

Then, they define the function

$$
d_{Y}(X, Z)=\min _{\left(X^{\prime}, Z^{\prime}\right) \in \mathcal{H}(X, Z)} d_{Y}^{\pi}\left(X^{\prime}, Z^{\prime}\right)
$$

and the set $\mathbf{Y}_{K}(X, Z)$ to be the set of $Y \in \mathbf{Y}$ such that $d_{Y}(X, Z)>K$.

For some (big enough) constant $K>0$, they define the projection complex $\mathcal{P}_{K}(\mathbf{Y})$ as a 1-complex whose vertex set is $\mathbf{Y}$ and such that there is an edge connecting two vertices $X$ and $Z$ if $\mathbf{Y}_{K}(X, Z)$ is empty.

Theorem 3.12 [1, Theorem 2.9] For $K$ sufficiently large $\mathcal{P}_{K}(\mathbf{Y})$ is a quasitree.

Also, suppose $G$ is a group acting on the set $\mathbf{Y}$, that there exists a function $d_{Y}^{\pi}$ satisfying (A1)-(A4) and projection distances are preserved, ie $d_{g(A)^{\pi}}(g(B), g(C))=d_{A}^{\pi}(B, C)$ for all $A, B, C \in \mathbf{Y}$ and $g \in G$. Then, $G$ acts naturally on $\mathcal{P}_{K}(\mathbf{Y})$. See Theorem 3.15.

Using this construction, several examples of groups which act on quasitrees are given in [1]. To obtain examples for Theorem 3.10 it suffices to check that there exist two independent hyperbolic elements $g_{1}, g_{2}$ such that $g_{1} \nsim g_{2}\left(\right.$ on $\left.P_{K}(\mathbf{Y})\right)$. 
In [1, Lemma 2.11], a technical lemma, there is certain constant $K^{\prime}$ involved which roughly depends on $K$. Fixing that constant and assuming $g \in G$ and $Y \in \mathbf{Y}$ such that

$$
d_{Y}\left(g^{-N}(Y), g^{N}(Y)\right)>K^{\prime}
$$

for some $N>0$ (see [1, Lemma 2.13]), they define the combinatorial axis as

$$
\mathbf{Y}_{K^{\prime}}(g)=\left\{Y \in \mathbf{Y} \mid d_{Y}\left(g^{-N}(Y), g^{N}(Y)\right)>K^{\prime} \text { for some } N>0\right\} .
$$

Also, fixing $K^{\prime}$, they consider the following axioms on $g \in G$ :

(B1) The element $g$ is contained in a unique maximal virtually cyclic subgroup, $\operatorname{EC}(g)$, the elementary closure of $g$.

(B2) $\mathrm{EC}(g)$ is malnormal, ie $\psi \mathrm{EC}(g) \psi^{-1}=\mathrm{EC}(g)$ for $\psi \in G$ implies $\psi \in \operatorname{EC}(g)$.

(B3) There is $Y \in \mathbf{Y}_{K^{\prime}}(g)$ and $m>0$ such that if $\psi \in G$ fixes $Y, g(Y), \ldots, g^{m}(Y)$, then $\psi \in \mathrm{EC}(g)$.

The following definition also comes from [1]. Assume $G$ is acting on a geodesic metric space $X$. Then, $g \in G$ is a WPD element if $\langle g\rangle$ has a bounded orbit in $X$ and for every $x \in X$ and $D>0$ there is $M>0$ such that the set

$$
\left\{\phi \in G \mid d\left(g^{i}(x), \phi\left(g^{i}(x)\right)\right) \leq D, i= \pm M\right\}
$$

is finite.

Proposition 3.13 [1, Proposition 2.16] Suppose $g \in G$ satisfies (B1)-B3. Then $g$ is a WPD element with respect to the action of $G$ on $\mathcal{P}_{K}(\mathbf{Y})$.

In [2] the authors define what they call weak proper discontinuity or WPD. A group action satisfies WPD if and only if

(i) there exists a hyperbolic element,

(ii) $G$ is not virtually cyclic,

(iii) every hyperbolic element in $G$ is a WPD element.

Then, they prove that if the action of a group $G$ on a $\delta$-hyperbolic graph $X$ satisfies WPD, then the action is nonelementary and there exist independent hyperbolic $g_{1}, g_{2}$ such that $g_{1} \not g_{2}$. See [2, Proposition 6].

However, in the proof of [2, Proposition 6(5)] the key is to find two independent WPD elements, more than having the condition on every hyperbolic element of the group. Therefore, this can be restated as follows. 
Proposition 3.14 Let $G$ be a group acting on a $\delta$-hyperbolic graph $X$ such that $G$ is not virtually cyclic and there exist two independent hyperbolic elements $f_{1}, f_{2} \in G$ which are WPD elements. Then there exist independent hyperbolic $g_{1}, g_{2}$ such that $g_{1} \not g_{2}$.

Theorem 3.15 [1, Theorem 2.17] Suppose a group $G$ acts on a set $\boldsymbol{Y}$ satisfying axioms (A1)-(A4) such that projection distances are preserved. Therefore, $G$ acts on the quasitree $\mathcal{P}_{K}(\mathbf{Y})$. Further, assume that there exist independent elements $g_{1}, g_{2} \in G$ that satisfy (B1)-(B3). Then, there is a nonabelian free subgroup $F \subset G$ all of whose nontrivial elements act on $\mathcal{P}_{K}(\mathbf{Y})$ hyperbolically as WPD elements.

Suppose then that there is a group $G$ and two independent elements $g_{1}, g_{2} \in G$ satisfying the conditions of the theorem. By Proposition 3.14 it follows that there are two independent hyperbolic elements $f_{1}, f_{2}$ such that $f_{1} \not f_{2}$, this is, the action of $G$ on $\mathbf{Y}$ is a Bestvina-Fujiwara action on a quasitree.

Hence, by Theorem 3.10:

Corollary 3.16 Suppose a group $G$ acts on a set $\boldsymbol{Y}$ satisfying axioms (A1)-(A4) such that projection distances are preserved. Further, assume that there exist independent elements $g_{1}, g_{2} \in G$ that satisfy (B1)-(B3). Then, there exists a bushy pseudocharacter $h: G \rightarrow \mathbb{R}$.

Example 3.17 Consider any nonelementary word hyperbolic group $G$. For every hyperbolic element $g \in G$, (B1)-(B2) hold. See Lück and Weiermann [11, Example 3.5] (or Theorem 3.2 in III. Г.3 on page 459 and Corollary 3.10 in III. Г.3 on page 462 from Bridson and Haefliger [4]). Also, the action may be chosen so (B3) holds.

For any nonelementary word hyperbolic group $G$ there is an action on a set $\mathbf{Y}$ satisfying axioms (A1)-(A4) so that projection distances are preserved (see [2, Examples 2.1]). Therefore, there exists a bushy pseudocharacter $h: G \rightarrow \mathbb{R}$.

\section{Quasiactions on trees}

Given a pseudocharacter $f: G \rightarrow \mathbb{R}$, Manning introduces the following constructions. The first one gives a tree obtained from the Cayley graph of the group.

Consider an (unambiguous) triangular generating set $S$. Then scale $f$ so that $f(G)$ misses $\mathbb{Z}+\frac{1}{2}$ and so that $f$ changes by at most $\frac{1}{4}$ over each edge. Let $\tilde{K}$ be the simply connected $2-$ complex obtained by attaching 2 -cells according to the relations 
of the presentation. Then, a tree is built with vertex set in one-to-one correspondence with the components of $\tilde{K} \backslash f^{-1}\left(\mathbb{Z}+\frac{1}{2}\right)$. The edges correspond to components of $f^{-1}\left(\mathbb{Z}+\frac{1}{2}\right)$, each of which is some possibly infinite track which separates $\tilde{K}$ into two components. This construction is also the starting point in the author's [14] where given a real valued function on a geodesic space we give a sufficient condition for the space to be quasi-isometric to a tree.

The next appears as [12, Definition 4.9].

Let $V$ be the set of components of $f^{-1}\left(\mathbb{Z}+\frac{1}{2}\right)$. Then $V$ is in one-to-one correspondence with the set of edges of $T$. Let $X$ be the simplicial graph with vertex set equal to $G \times V$ and the following edge condition: Two distinct vertices $(g, \tau)$ and $\left(g^{\prime}, \tau^{\prime}\right)$ are to be connected by an edge if there is some $h$ so that $h g(\tau)$ and $h g^{\prime}\left(\tau^{\prime}\right)$ are contained in the same connected component of $f^{-1}\left[n-\frac{3}{2}, n+\frac{1}{2}\right]$ for some $n$. The zero-skeleton $X^{0}$ is endowed with a $G$-action by setting $g\left(g_{0}, \tau_{0}\right)=\left(g g_{0}, \tau_{0}\right)$. Since this action respects the edge condition on pairs of vertices, it extends to an action on $X$. We will refer to this particular one as Manning's action.

Proposition 4.1 [12, Proposition 4.27] If $f: G \rightarrow \mathbb{R}$ is a bushy pseudocharacter, then Manning's action is a Bestvina-Fujiwara action.

Theorem 4.2 [12, Theorem 4.15] The space $X$ satisfies the Bottleneck Property.

Therefore, from Theorem 3.10, we can give the following corollary which would be some kind of converse to Proposition 4.1.

Corollary 4.3 If Manning's action is a Bestvina-Fujiwara action, then there is a bushy pseudocharacter $h: G \rightarrow \mathbb{R}$.

Lemma 4.4 [12, Lemma 4.17] There is an injective map from $E(f)$ to $\partial X$.

Theorem 4.5 [12, Theorem 4.20] If $f: G \rightarrow \mathbb{R}$ is a pseudocharacter which is not uniform, then $G$ admits a cobounded quasiaction on a bushy tree.

Then, it is readily seen, from the construction of the action and the bushy tree, that Corollary 2.11 yields the following.

Corollary 4.6 Consider a nonelementary action of a group $G$ on a quasitree $X$. If the action is metrically proper, then for any pseudocharacter $h: G \rightarrow \mathbb{R}$ and any pair of independent $g_{1}, g_{2} \in G$ so that $h\left(g_{1}\right)>0$ and $h\left(g_{2}\right)>0$, there is a cobounded quasiaction of $G$ on a bushy tree $T$ so that there is an injective map from $E(h)$ to $\partial T$. 


\section{Space of pseudocharacters}

Given a group $G$, quasicharacters and pseudocharacters are major tools in the study of the bounded cohomology group $H_{b}^{2}(G ; \mathbb{R})$ as we can see in [2].

The bounded cohomology group $H_{b}^{*}(G ; \mathbb{R})$ of a discrete group $G$ is defined by the cochain complex $C_{b}^{k}(G ; \mathbb{R})$, where

$$
C_{b}^{k}(G ; \mathbb{R})=\left\{f: G^{k} \rightarrow \mathbb{R}\left|\sup _{G^{k}}\right| f\left(g_{1}, \ldots, g_{k}\right) \mid<\infty\right\}
$$

and the boundary $\delta: C_{b}^{k}(G ; A) \rightarrow C_{b}^{k+1}(G ; A)$ is given by

$$
\begin{aligned}
\delta f\left(g_{0}, \ldots, g_{k}\right)=f\left(g_{1}, \ldots, g_{k}\right)+ & \sum_{i=1}^{k}(-1)^{i} f\left(g_{0}, \ldots, g_{i-1} g_{i}, \ldots, g_{k}\right) \\
& +(-1)^{k+1} f\left(g_{0}, \ldots, g_{k-1}\right) .
\end{aligned}
$$

See Gromov [8] and Ivanov [10] as general references for bounded cohomology.

Remark Note that 1 -cocycles are just group homomorphisms $f: G \rightarrow \mathbb{R}$. In fact, $\operatorname{Hom}(G)=H^{1}(G ; \mathbb{R})$. A quasicharacter is an element $f \in C^{1}(G ; \mathbb{R})$ whose coboundary $\delta f$ lies in $C_{b}^{2}(G ; \mathbb{R})$. A pseudocharacter is a quasicharacter such that $f\left(g^{k}\right)=k f(g)$ for all $k \in \mathbb{Z}$ and $g \in G$.

Let $\mathcal{V}(G)$ be the vector space of all quasihomomorphisms $G \rightarrow \mathbb{R}$ and let $\operatorname{BDD}(G)$ be the subspace of all bounded functions. Then, let $\mathrm{QH}(G)=\mathcal{V}(G) / \operatorname{BDD}(G)$.

There is an exact sequence

$$
0 \rightarrow H^{1}(G ; \mathbb{R}) \rightarrow \mathrm{QH}(G) \rightarrow H_{b}^{2}(G ; \mathbb{R}) \rightarrow H^{2}(G ; \mathbb{R})
$$

Using the sequence $f_{1}, f_{2}, \ldots$ obtained in Proposition 3.8, Bestvina and Fujiwara prove that $\left[h_{i}\right] \in \mathrm{QH}(G)$ is not a linear combination of $\left[h_{1}\right], \ldots,\left[h_{i-1}\right]$, ie, the sequence $\left[h_{i}\right]$ consists of linearly independent elements (see the proof of [2, Theorem 1]). This implies that the dimension of $H_{b}^{2}(G ; \mathbb{R})$ as a vector space over $\mathbb{R}$ is the cardinal of the continuum. See [6, Corollary 1.3] and [2, Theorem 1].

Therefore, since the argument in Theorem 3.10 works also for any pair $f_{i}, f_{i+1}$, the following is immediate.

Corollary 5.1 Let $G$ be a group acting on a (geodesic) Gromov hyperbolic graph $X$ satisfying the bottleneck property. If it is a Bestvina-Fujiwara action, then the dimension of the subspace generated by the bushy pseudocharacters as a vector space over $\mathbb{R}$ is the cardinal of the continuum. 
Corollary 5.2 If a Cayley graph $X=\Gamma(G, S)$ satisfies the bottleneck property and the canonical action of the group is a Bestvina-Fujiwara action, then the dimension of the subspace generated by the bushy pseudocharacters as a vector space over $\mathbb{R}$ is the cardinal of the continuum.

Corollary 5.3 If Manning's action is a Bestvina-Fujiwara action, then the dimension of the subspace generated by the bushy pseudocharacters on $G$ as a vector space over $\mathbb{R}$ is the cardinal of the continuum.

In particular:

Corollary 5.4 If there is a bushy pseudocharacter $h: G \rightarrow \mathbb{R}$ then the dimension of the subspace generated by the bushy pseudocharacters on $G$ as a vector space over $\mathbb{R}$ is the cardinal of the continuum.

This, together with Corollary 2.14 and Corollary 2.15 yields:

Corollary 5.5 Consider a nonelementary action of a group $G$ on a quasitree $X$ and a nonelementary pseudocharacter $h: G \rightarrow \mathbb{R}$. Then, if $h$ is bornologous on the action, the dimension of the subspace generated by the bushy pseudocharacters on $G$ (in particular, the dimension of $H_{b}^{2}(G ; \mathbb{R})$ ) as a vector space over $\mathbb{R}$ is the cardinal of the continuum.

Corollary 5.6 Consider a nonelementary action of a group $G$ on a quasitree $X$. If the action is metrically proper and there exist a nonelementary pseudocharacter then the dimension of the subspace generated by the bushy pseudocharacters on $G$ (in particular, the dimension of $H_{b}^{2}(G ; \mathbb{R})$ ) as a vector space over $\mathbb{R}$ is the cardinal of the continuum.

\section{References}

[1] M Bestvina, K Bromberg, K Fujiwara, Asymptotic dimension of mapping class groups is finite arXiv:1006.1939v2

[2] M Bestvina, K Fujiwara, Bounded cohomology of subgroups of mapping class groups, Geom. Topol. 6 (2002) 69-89 MR1914565

[3] B H Bowditch, Notes on Gromov's hyperbolicity criterion for path-metric spaces, from: "Group theory from a geometrical viewpoint (Trieste, 1990)", (É Ghys, A Haefliger, A Verjovsky, editors), World Sci. Publ., River Edge, NJ (1991) 64-167 MR1170364

[4] M R Bridson, A Haefliger, Metric spaces of non-positive curvature, Grundl. Math. Wissen. 319, Springer, Berlin (1999) MR1744486 
[5] D B A Epstein, K Fujiwara, The second bounded cohomology of word-hyperbolic groups, Topology 36 (1997) 1275-1289 MR1452851

[6] K Fujiwara, The second bounded cohomology of a group acting on a Gromovhyperbolic space, Proc. London Math. Soc. 76 (1998) 70-94 MR1476898

[7] K Fujiwara, The second bounded cohomology of an amalgamated free product of groups, Trans. Amer. Math. Soc. 352 (2000) 1113-1129 MR1491864

[8] M Gromov, Volume and bounded cohomology, Inst. Hautes Études Sci. Publ. Math. (1982) 5-99 MR686042

[9] M Gromov, Hyperbolic groups, from: "Essays in group theory", (S M Gersten, editor), Math. Sci. Res. Inst. Publ. 8, Springer, New York (1987) 75-263 MR919829

[10] N V Ivanov, Foundations of the theory of bounded cohomology, Zap. Nauchn. Sem. Leningrad. Otdel. Mat. Inst. Steklov. (LOMI) 143 (1985) 69-109, 177-178 MR806562

[11] W Lück, M Weiermann, On the classifying space of the family of virtually cyclic subgroups, PAMQ 8 (2012) 497-555

[12] J F Manning, Geometry of pseudocharacters, Geom. Topol. 9 (2005) 1147-1185 MR2174263

[13] J F Manning, Quasi-actions on trees and property (QFA), J. London Math. Soc. 73 (2006) 84-108 MR2197372 With an appendix by N Monod and B Rémy

[14] A Martínez-Pérez, Real valued functions and metric spaces quasi-isometric to trees arXiv: 1103.5908

[15] L Mosher, M Sageev, K Whyte, Quasi-actions on trees I. Bounded valence, Ann. of Math. 158 (2003) 115-164 MR1998479

Departamento de Análisis Económico y Finanzas, Universidad de Castilla-La Mancha Avda Real Fábrica de Seda, s/n, 45600 Talavera de la Reina, Toledo, Spain alvaro.martinezperez@uclm.es

Received: 20 July 2011 Revised: 17 April 2012 